\title{
Resistência de biótipos de arroz-vermelho aos herbicidas imazapyr + imazapic e alternativas de controle ${ }^{1}$
}

\author{
Rafael Salles Rubin', Dirceu Agostinetto ${ }^{3}$, Roberta Manica-Berto ${ }^{4}$, \\ Diego Severo Fraga ${ }^{5}$, Camila Peligrinotti Tarouco ${ }^{5}$ \\ http://dx.doi.org/10.1590/0034-737X201461050009
}

\begin{abstract}
RESUMO
O uso intenso de herbicidas com o mesmo mecanismo de ação na cultura do arroz (Oryza sativa L.) tem selecionado biótipos resistentes, como, por exemplo, o arroz-vermelho (planta daninha) resistente aos herbicidas imidazolinonas. Por essa razão, este trabalho foi desenvolvido com o objetivo de avaliar a resistência de arroz-vermelho ao herbicida imazapyr + imazapic, na região sul do Rio Grande do Sul, e o controle do biótipo resistente de arroz-vermelho com os herbicidas alternativos clethodim e glyphosate. Foram realizados três experimentos, em delineamento casualizado, arranjados em esquema fatorial. No primeiro experimento, o fator A testou biótipos de arroz-vermelho [ORYSA 184 (resistente) e ORYSA 188 (susceptível)], o fator B comparou herbicidas (imazapyr + imazapic, clethodim e glyphosate) e o fator $\mathrm{C}$ avaliou doses dos herbicidas $(0 ; 0,5 ; 1 ; 2 ; 4 ; 8 ; 16$ e 32 vezes a dose recomendada). No segundo, compararam-se biótipos de arroz-vermelho e doses do herbicida imazapyr + imazapic $(0 ; 0,5 ; 1 ; 2 ; 4 ; 8 ; 16 ; 32$ e 64 vezes a dose recomendada). No terceiro, testaram-se biótipos de arroz-vermelho e doses diferentes do herbicida imazapyr + imazapic para cada biótipo. O biótipo ORYSA 184 é resistente ao imazapyr + imazapic, quando aplicada a dose máxima de registro e estádio indicado. Os herbicidas clethodim e glyphosate, detentores de mecanismos de ação alternativos, controlam o biótipo resistente ORYSA 184 de arroz-vermelho.
\end{abstract}

Palavras-chave: Oryza sativa, imidazolinonas, controle químico.

\section{ABSTRACT}

\section{Resistance of biotypes of red rice to imazapyr + imazapic herbicides and control alternatives}

The intenseuse of herbicides with the same action mechanism in rice (Oryza sativa L.) has selected resistant biotypes, such as red rice (weed) resistant to imidazolinone herbicides. Therefore, the objective of this work was to evaluate the resistance of red rice to the imazapyr + imazapic herbicide in the southern region of Rio Grande of Sul, Brazil, and the control of the red rice resistant biotype with the alternative herbicides, clethodim and glyphosate. Three experiments were carried out in a completely randomized design, arranged in a factorial design. In the first experiment, the factor A tested biotypes of red rice [ORYSA 184 (resistant) and ORYSA 188 (susceptible)], factor B compared herbicides (imazapyr + imazapic, clethodim and glyphosate) and factor C evaluated dosages of herbicide $(0,0.5,1,2,4,8,16$ and 32 times the recommended dosage). The second experiment compared biotypes of red rice and dosages of the imazapyr + imazapicherbicide $(0,0.5,1,2,4,8,16,32$ and 64 times the recommended dosages).

\footnotetext{
Recebido para publicação em 21/01/2013 e aprovado em 08/10/2013.

1 Extraído da Dissertação de Mestrado em Fitossanidade do primeiro autor.

${ }^{2}$ Engenheiro-Agrônomo. Mestre, Universidade Federal de Pelotas, Faculdade de Agronomia Eliseu Maciel, Departamento de Fitossanidade, Campus Universitário s/nº $96010-900$, Caixa Postal, 354, Pelotas, RS, Brasil. E-mail: rsallesr@ hotmail.com (Autor para correspondência)

${ }^{3}$ Engenheiro-Agrônomo. Doutor, Professor Adjunto, Universidade Federal de Pelotas, Faculdade de Agronomia Eliseu Maciel, Departamento de Fitossanidade, Campus Universitário $\mathrm{s} / \mathrm{n}^{\circ}, 96010-900$, Caixa Postal, 354, Pelotas, RS, Brasil. E-mail: agostinetto@ig.com.br

${ }^{4}$ Engenheira- Agrônoma. Pós-Doutoranda, Universidade Federal de Pelotas, Faculdade de Agronomia Eliseu Maciel, Departamento de Fitossanidade, Campus Universitário s/no 96010-900, Caixa Postal, 354, Pelotas, RS, Brasil. E-mail: robertamanica@yahoo.com.br

${ }^{5}$ Engenheiro-Agrônomo. Mestre. Departamento de Fitossanidade. Universidade Federal de Pelotas, Faculdade de Agronomia Eliseu Maciel, Campus Universitário s/nº $96010-900$, Caixa Postal,354, Pelotas, RS, Brasil. fragadiegos@gmail.com; milatarouco@gmail.com
} 
The third experiment tested biotypes of red rice and different doses of the imazapyr + imazapic for each biotype. The biotype ORYSA 184 is resistant to imazapic + imazapyr when applied at the maximum indicated record and stadium dose. The herbicides glyphosate and clethodim, holders of alternative mechanisms of action, control the red rice resistant biotype ORYSA 184.

Key words:chemical control, imidazolinone, Oryza sativa.

\section{INTRODUÇÃO}

A ocorrência de plantas daninhas é um dos principais fatores limitantes do potencial produtivo da cultura do arroz irrigado, sendo as perdas variáveis em razão de características da espécie infestante e das práticas de manejo adotadas na lavoura (Fleck et al., 2008). Dentre as plantas daninhas que ocorrem na lavoura orizícola, destaca-se o arroz-vermelho (Oryza sativa L.), por ser a que mais limita o potencial de produtividade do arroz (Shivrain et al., 2009).

Por pertencer à mesma espécie do arroz cultivado, o arroz-vermelho não pode ser controlado por herbicidas em situações de utilização de cultivares não transgênicas, ou não selecionadas para tolerância a herbicidas. Entretanto, foram desenvolvidas plantas de arroz resistentes a herbicidas pertencentes ao grupo químico das imidazolinonas, tendo-se, então, alternativas de controle químico do arroz-vermelho (Croughan et al., 1996; Steele et al., 2002).

O mecanismo de ação desses herbicidas é a inibição da atividade das enzimas acetolactato sintase (ALS), ou aceto-hidroxi sintase (AHAS), na rota de síntese dos aminoácidos de cadeia ramificada, como valina, leucina e isoleucina (Rizzardi et al., 2004). Os herbicidas inibidores de ALS são muito utilizados na agricultura, principalmente por apresentarem elevada eficácia no controle de plantas daninhas, serem utilizados em baixas doses e por serem altamente seletivos às culturas (Vidal \& Merotto Júnior, 2001).

A resistência de plantas daninhas a herbicidas provoca, na maioria dos casos, alterações nos biótipos resistentes, em comparação com o biótipo susceptível. Dentre elas, encontra-se a dose necessária para controlar $50 \%$ da população $\left(\mathrm{C}_{50}\right)$ e reduzir $50 \%$ da produção de matéria da parte aérea seca $\left(\mathrm{MS}_{50}\right)$. Os valores da $\mathrm{C}_{50}$ para herbicidas inibidores de ALS foram de 466,6 e 11,4 $\mathrm{g} \mathrm{ha}^{-1}$, para Bidens pilosa (Christoffoleti, 2002) e de 136,3, e 5,5 $\mathrm{g} \mathrm{ha}^{-1}$, para $B$. subalternans, resistente e susceptível a chlorimuron-ethyl, respectivamente (López-Ovejero et al., 2006). Já, para o herbicida imazethapyr, os valores da $\mathrm{C}_{50}$ observados foram de 4402 e $77 \mathrm{~g} \mathrm{ha}^{-1}$, para B. pilosa (Christoffoleti, 2002); de 146,7 e 13,7 $\mathrm{g} \mathrm{ha}^{-1}$, para B. subalternans (López-Ovejero et al., 2006), e de 239,3 e 30,5 $\mathrm{g} \mathrm{ha}^{-1}$, para arroz-vermelho (Dornelles et al., 2010), resistentes e susceptíveis, respectivamente.
Com relação a $\mathrm{MS}_{50}$, para B. subalternans resistente e susceptível aos herbicidas chlorimuron-ethyl, imazethapyr e cloransulan foram observados valores de 28,9 e $0,08 \mathrm{~g} \mathrm{ha}^{-1} ; 974,2$ e $1,53 \mathrm{~g} \mathrm{ha}^{-1}$; e, 585,9 e 1,52 g ha $^{-1}$, respectivamente (Lamego et al., 2009). Em relação ao herbicida imazethapyr, os valores de $\mathrm{MS}_{50}$ encontrados para biótipos de arroz-vermelho resistente e susceptível foram de 176,9 e $56,5 \mathrm{~g} \mathrm{ha}^{-1}$, respectivamente (Dornelles et al., 2010).

Os biótipos resistentes necessitam de doses maiores que as utilizadas, tanto para controlar quanto para reduzir o crescimento dos biótipos susceptíveis. Deste modo, torna-se importante indicar alternativas de controle químico para as espécies resistentes a herbicida, com finalidade de reduzir a população destas plantas para níveis em que não tragam prejuízos ao produtor.

Por essa razão, este trabalho foi desenvolvido com o objetivo de avaliar a resistência de arroz-vermelho ao herbicida imazapyr + imazapic, na região sul do Rio Grande do Sul, e o controle do biótipo resistente de arrozvermelho com os herbicidas alternativos clethodim e glyphosate.

\section{MATERIAL E MÉTODOS}

Para caracterizar a resistência de arroz-vermelho ao herbicida imazapyr + imazapic, foram desenvolvidos três experimentos, em casa de vegetação, pertencente ao Departamento de Fitossanidade/FAEM/UFPel. Foram coletadas e identificadas sementes de arroz-vermelho com suspeita de resistência aos herbicidas inibidores da enzima ALS e, também, sementes em área que nunca recebeu tratamento com esses herbicidas (testemunha susceptível). O biótipo com suspeita de resistência (ORYSA 184) e o biótipo susceptível (ORYSA 188) foram coletados nos municípios de Capão do Leão (52 $41^{\circ}$ '52" O e 31 $54^{\circ}$ ' 23" S) e Rio Grande $\left(52^{\circ} 31^{\prime} 04^{\prime \prime} \mathrm{O}\right.$ e $\left.32^{\circ} 17^{\prime} 12^{\prime \prime} \mathrm{S}\right)$, respectivamente, na região sul do Estado do Rio Grande do Sul.

Cada unidade experimental foi composta por vaso plástico com capacidade para $5 \mathrm{~L}$, contendo solo classificado como Planossolo Hidromórfico eutrófico solódico, pertencente à unidade de mapeamento Pelotas (Embrapa-CNPS, 2006). Foram distribuídas 15 sementes em cada recipiente e, após a emergência das plantas, foi procedido o desbaste, deixando-se cinco plantas por vaso. A adubação foi realiza- 
da na instalação do experimento, obedecendo à análise química do solo. A aplicação dos tratamentos foi realizada em pós-emergência, quando as plantas de arroz-vermelho atingiram quatro folhas, com pulverizador costal, pressurizado a $\mathrm{CO}_{2}$, equipado com bico do tipo leque com ponta de pulverização 110.015 , calibrado para aplicar $150 \mathrm{~L} \mathrm{ha}^{-1}$ de calda herbicida.

No primeiro experimento, os tratamentos foram dispostos em delineamento experimental completamente casualizado, com quatro repetições, em esquema fatorial. O fator A testou biótipos de arroz-vermelho (ORYSA 184 e ORYSA 188), o fator B comparou herbicidas (imazapyr + imazapic, clethodim e glyphosate) e o fator C avaliou doses dos herbicidas. As doses testadas para os biótipos equivaleram às proporções $0 ; 0,5 ; 1 ; 2 ; 4 ; 8 ; 16$ e 32 vezes a dose recomendada dos herbicidas imazapyr + imazapic $\left(73,5\right.$ g i.a. ha ${ }^{-1}+24,5$ g i.a. ha $\left.a^{-1}\right)$; clethodim $\left(84\right.$ g i.a.. ha $\left.{ }^{-1}\right)$; e, glyphosate (720 g e.a ha ${ }^{-1}$ ) (Agrofit, 2012). Foi adicionado ao herbicida imazapyr + imazapic o adjuvante $\operatorname{Dash}^{\circledR}$ a $0,5 \% \mathrm{v} / \mathrm{v}$ e ao clethodim, Assist ${ }^{\circledR}$ a $1 \% \mathrm{v} / \mathrm{v}$.

$\mathrm{O}$ segundo experimento seguiu o mesmo delineamento experimental, porém, o fator A comparou os biótipos de arroz-vermelho e o fator B testou as doses do herbicida imazapyr + imazapic, as quais equivaleram às proporções $0 ; 0,5 ; 1 ; 2 ; 4 ; 8 ; 16 ; 32$; e, 64 vezes a dose recomendada do herbicida $\left(73,5 \mathrm{~g}\right.$ i.a. $\mathrm{ha}^{-1}+24,5 \mathrm{~g}$ i.a. ha $\left.{ }^{-1}\right)$ (Agrofit, 2012).

O terceiro experimento foi conduzido seguindo o mesmo delineamento que os anteriores, sendo atribuídos ao fator $\mathrm{A}$ os biótipos de arroz-vermelho e, ao fator B, as doses do herbicida imazapyr + imazapic. As doses testadas para o biótipo resistente equivaleram às proporções $0 ; 0,5 ; 1 ; 2 ; 4$; $8 ; 16 ; 32 ;$ e, 64 vezes a dose recomendada e, para o biótipo susceptível, a proporção foi de $0 ; 0,0625 ; 0,125 ; 0,25 ; 0,5 ; 1$; 2; e, 4 vezes a dose recomendada do herbicida (Agrofit, 2012). Em ambos os experimentos foi adicionado ao herbicida o adjuvante Dash $^{\circledR}$ a $0,5 \%$ v/v.

As variáveis avaliadas foram controle visual e matéria seca da parte aérea (MSPA) aos 28 dias após a aplicação dos tratamentos (DAT), adotando-se a escala percentual, em que zero $(0)$ e cem (100) corresponderam à ausência de dano e à morte de todas as plantas, respectivamente. A MSPA foi determinada pela secagem do material vegetal, em estufa, a temperatura de $60^{\circ} \mathrm{C}$, até atingir massa constante.

Os dados foram analisados quanto à normalidade pelo teste de Shapiro-Wilk e, à homocedasticidade, pelo teste de Hartley e, posteriormente, submetidos à análise de variância $(p \leq 0,05)$. Em caso de significância, os efeitos de biótipos foram analisados pelo teste $\mathrm{t}(\mathrm{p} \leq 0,05)$, os de herbicidas pelo teste de Tukey $(\mathrm{p} \leq 0,05)$ e os de dose por modelo de regressão não linear $(\mathrm{p} \leq 0,05)$, representado pela equação 1 , para níveis de controle, e pela equação 2 , para acúmulo de MSPA, para o primeiro e segundo experimen- tos; e, pela equação 3, para níveis de controle e MSPA do terceiro experimento:

$\mathrm{y}=\mathrm{a}\left(1-\mathrm{e}^{-\mathrm{bx}}\right)$

Equação 1

em que: $a=$ valor máximo estimado para a variável resposta; $b=$ inclinação da curva; $x=\mathrm{x}$ a dose recomendada dos herbicidas imazapyr + imazapic, clethodim e glyphosate $\left(\mathrm{g} \mathrm{ha}^{-1}\right) ; e=$ constante;

$\mathrm{y}=\mathrm{y}_{\mathrm{o}}+\mathrm{a}\left(1-\mathrm{e}^{-\mathrm{bx}}\right)$

Equação 2

onde: $\mathrm{y}_{\mathrm{o}}=$ mínima matéria seca da parte aérea $(\mathrm{g}) ; a=$ valor máximo estimado para a variável resposta; $b=$ inclinação da curva; $x=\mathrm{x}$ a dose recomendada dos herbicidas imazapyr + imazapic, clethodim e glyphosate $\left(\mathrm{g} \mathrm{ha}^{-1}\right) ; e=$ constante;

$\mathrm{y}=\mathrm{a} /\left[1+\left(\mathrm{x} / \mathrm{x}_{0}\right)^{\mathrm{b}}\right]$

Equação 3

em que: $y=$ percentagem de controle ou gramas de matéria seca da parte aérea; $x=\mathrm{x}$ a dose recomendada do herbicida; e $a, x_{0}$ e $b=$ parâmetros da equação, sendo $a$ a diferença entre os pontos máximo e mínimo da curva, $x_{0}$ é a dose que proporciona $50 \%$ de resposta da variável e $b$ a declividade da curva.

$\mathrm{A} \mathrm{C}_{50}$ e $\mathrm{MS}_{50}$ foram calculadas com base no modelo (equações 1 e 2, respectivamente), pela estimativa para o valor de $x$, em que o $y$ resultasse no valor de $50 \%$. Para o terceiro experimento, os valores da $\mathrm{C}_{50} \mathrm{e} \mathrm{MS}_{50}$ foram calculados pelo modelo de regressão log-logístico não linear (Seefeldt et al., 1995). Como nesse experimento as doses testadas foram diferentes para o biótipo resistente, em relação às doses para o susceptível, $\mathrm{a}_{50} \mathrm{e} \mathrm{MS}_{50}$ foram calculadas pelos intervalos de confiança entre doses, a $95 \%$, os quais permitiram a comparação entre os biótipos testados.

O fator de resistência (FR) foi calculado pela divisão da $\mathrm{C}_{50}$ ou $\mathrm{MS}_{50}$ do biótipo resistente pelos correspondentes ao do biótipo susceptível. O FR expressa o número de vezes em que a dose necessária para controlar $50 \%$ do biótipo resistente é superior à dose que controla 50\% do biótipo susceptível (Hall et al., 1998).

\section{RESULTADOS E DISCUSSÃO}

No primeiro experimento, observou-se interação dos fatores estudados para todas as variáveis. Em geral, não se verificaram diferenças no controle dos biótipos de arroz-vermelho pelos herbicidas clethodim ou glyphosate (Tabela 1). Já, o herbicida imazapyr + imazapic apresentou maior controle sobre o biótipo susceptível, em comparação com o controle sobre o biótipo suspeito de resistência, em todas as doses testadas.

A comparação entre herbicidas para o biótipo suspeito de ser resistente demonstrou, em todas as doses 
testadas, que os herbicidas clethodim ou glyphosate foram superiores, em relação ao controle, ao imazapyr + imazapic (Tabela 1). Por sua vez, para o biótipo susceptível, não se verificaram diferenças entre herbicidas, tendo sido observado controle superior a $95 \%$ mesmo na menor dose testada.

Para os herbicidas clethodim ou glyphosate, verificouse controle crescente, à medida que a dose foi aumentada para ambos os biótipos estudados (Tabela 1). Com relação ao herbicida imazapyr + imazapic, observou-se controle deficiente para o biótipo com suspeita de resistência, sendo o valor máximo estimado pelo modelo para a maior dose testada (32 vezes) de aproximadamente 57\%, enquanto para o biótipo susceptível foi próximo a $99 \%$.

Com relação à MSPA, em geral, não foram observadas diferenças entre os biótipos quando da aplicação do herbicida clethodim (Tabela 1). Para o herbicida glyphosate, verificou-se maior produção de MSPA, para o biótipo resistente, nas doses superiores à dose de registro, com exceção de quatro vezes a dose. O biótipo resistente apresentou maiores valores de MSPA, em relação aos do biótipo susceptível, para todas as doses testadas do herbicida imazapyr + imazapic, com exceção da maior dose.

Para o biótipo resistente, constatou-se que as plantas submetidas à aplicação dos herbicidas clethodim ou glyphosate apresentaram, em comparação com as submetidas à imazapyr + imazapic, menor produção de MSPA, em consequência do maior controle propiciado (Tabela 1). Já, para o biótipo susceptível, em todas as doses, não foram observadas diferenças, em relação à MSPA, para os herbicidas testados.

Para o efeito de doses, os herbicidas clethodim e glyphosate apresentaram drástica redução da produção de MSPA, a partir da primeira dose de herbicida testada (Tabela 1). Para o herbicida imazapyr + imazapic, consi-

Tabela 1. Controle (\%) e matéria seca da parte aérea - MSPA (g) de biótipos de arroz-vermelho resistente (RES) e susceptível (SUS) ao herbicida imazapyr + imazapic, em função da aplicação de doses dos herbicidas clethodim (CLE), glyphosate (GLY) e imazapyr + imazapic (IMA), aos 28 dias após a aplicação dos tratamentos. FAEM/UFPel, Capão do Leão/RS, 2010/11

\begin{tabular}{|c|c|c|c|c|c|c|}
\hline \multicolumn{4}{|c|}{ Resistente } & \multicolumn{3}{|c|}{ Susceptível } \\
\hline Dose $^{1}$ & Clethodim & Glyphosate & $\begin{array}{c}\text { Imazapyr } \\
\text { +Imazapic }\end{array}$ & Clethodim & Glyphosate & $\begin{array}{c}\text { Imazapyr } \\
\text { + Imazapic }\end{array}$ \\
\hline \multicolumn{7}{|c|}{ Controle (\%) } \\
\hline 0 & $0,0 \mathrm{a}^{\mathrm{ns}}$ & $0,0 a^{\text {ns }}$ & 0,0 a ns & $0,0 \mathrm{a}$ & $0,0 \mathrm{a}$ & $0,0 \mathrm{a}$ \\
\hline 0,5 & $78,0 \mathrm{~b}^{\mathrm{ns}}$ & $97,7 \mathrm{a} *$ & $22,0 \mathrm{c} *$ & $95,5 \mathrm{a}$ & $100,0 \mathrm{a}$ & $96,2 \mathrm{a}$ \\
\hline 1 & $95,0 a^{\mathrm{ns}}$ & $100,0 a^{\mathrm{ns}}$ & $42,2 \mathrm{~b} *$ & $98,0 \mathrm{a}$ & $100,0 \mathrm{a}$ & $96,8 \mathrm{a}$ \\
\hline 2 & $99,0 a^{\text {ns }}$ & $100,0 a^{\mathrm{ns}}$ & $49,5 \mathrm{~b} *$ & $100,0 \mathrm{a}$ & $99,5 \mathrm{a}$ & $97,2 \mathrm{a}$ \\
\hline 4 & $100,0 a^{\mathrm{ns}}$ & $100,0 a^{\mathrm{ns}}$ & $36,3 \mathrm{~b} *$ & $100,0 \mathrm{a}$ & $100,0 \mathrm{a}$ & $98,7 \mathrm{a}$ \\
\hline 8 & $100,0 a^{\mathrm{ns}}$ & $100,0 a^{\mathrm{ns}}$ & $64,5 \mathrm{~b} *$ & $100,0 \mathrm{a}$ & $100,0 \mathrm{a}$ & $99,2 \mathrm{a}$ \\
\hline 16 & $100,0 a^{\mathrm{ns}}$ & $100,0 a^{\mathrm{ns}}$ & $68,2 \mathrm{~b} *$ & $100,0 \mathrm{a}$ & $100,0 \mathrm{a}$ & $99,5 \mathrm{a}$ \\
\hline 32 & $100,0 a^{\mathrm{ns}}$ & $100,0 a^{\text {ns }}$ & $64,2 b *$ & $100,0 \mathrm{a}$ & $100,0 \mathrm{a}$ & $100,0 \mathrm{a}$ \\
\hline \multicolumn{7}{|c|}{$\operatorname{MSPA}(\mathrm{g})$} \\
\hline 0 & $18,19 a^{n s}$ & $17,21 \mathrm{a} *$ & $17,84 a^{\mathrm{ns}}$ & $19,57 \mathrm{a}$ & $20,75 \mathrm{a}$ & $15,45 \mathrm{~b}$ \\
\hline 0,5 & $4,24 \mathrm{~b} *$ & $1,26 \mathrm{c}^{\mathrm{ns}}$ & $15,69 \mathrm{a} *$ & $1,26 \mathrm{a}$ & $1,02 \mathrm{a}$ & $1,43 \mathrm{a}$ \\
\hline 1 & $1,34 \mathrm{~b}^{\mathrm{ns}}$ & $0,86 b^{n s}$ & $12,88 \mathrm{a} *$ & $1,27 \mathrm{a}$ & $0,83 \mathrm{a}$ & $1,40 \mathrm{a}$ \\
\hline 2 & $1,64 \mathrm{~b}^{\mathrm{ns}}$ & $0,82 \mathrm{~b} *$ & $10,36 \mathrm{a} *$ & $1,26 \mathrm{a}$ & $0,44 \mathrm{a}$ & $1,64 \mathrm{a}$ \\
\hline 4 & $1,15 \mathrm{~b}^{\mathrm{ns}}$ & $0,39 b^{n s}$ & $13,06 \mathrm{a} *$ & $1,24 \mathrm{a}$ & $0,34 \mathrm{a}$ & $1,20 \mathrm{a}$ \\
\hline 8 & $1,18 \mathrm{~b}^{\mathrm{ns}}$ & $0,79 \mathrm{~b} *$ & $6,80 \mathrm{a} *$ & $0,89 \mathrm{a}$ & $0,33 \mathrm{a}$ & $1,27 \quad \mathrm{a}$ \\
\hline 16 & $0,67 b^{\mathrm{ns}}$ & $0,54 \mathrm{~b} *$ & $5,47 \mathrm{a} *$ & $0,77 \mathrm{a}$ & $0,32 \mathrm{a}$ & $1,26 \mathrm{a}$ \\
\hline 32 & $0,71 b^{\text {ns }}$ & $0,51 \mathrm{~b}$ & $* 3,47 \mathrm{a}^{\mathrm{ns}}$ & $0,89 \mathrm{a}$ & $0,31 \mathrm{a}$ & $1,02 \mathrm{a}$ \\
\hline \multicolumn{2}{|c|}{ Equações (Controle) ${ }^{2}$} & $\mathbf{R}^{2}$ & \multicolumn{3}{|c|}{ Equações (MSPA) } & $\mathbf{R}^{2}$ \\
\hline \multicolumn{2}{|c|}{$\mathrm{y}($ CLE-RES $)=99,84\left(1-\mathrm{e}^{-3,03 \mathrm{x}}\right)$} & 1,00 & \multicolumn{3}{|c|}{$\mathrm{y}($ CLE-RES $)=18,20-17,17\left(1-\mathrm{e}^{-3,41 x}\right)$} & 1,00 \\
\hline \multicolumn{2}{|c|}{$\mathrm{y}($ CLE-SUS $)=99,63\left(1-\mathrm{e}^{-6,30 \mathrm{x}}\right)$} & 1,00 & \multicolumn{3}{|c|}{$\mathrm{y}(\mathrm{CLE}-\mathrm{SUS})=19,57-18,52\left(1-\mathrm{e}^{-8,88 x}\right)$} & 1,00 \\
\hline \multicolumn{2}{|c|}{$y($ GLY-RES $)=100,00\left(1-e^{-7,58 x}\right)$} & 1,00 & \multicolumn{3}{|c|}{$y($ GLY-RES $)=17,21-16,56\left(1-\mathrm{e}^{-6,54 x}\right)$} & 1,00 \\
\hline \multicolumn{2}{|c|}{$y($ GLY-SUS $)=100,00\left(1-\mathrm{e}^{-45,25 x}\right)$} & 1,00 & \multicolumn{3}{|c|}{$y($ GLY-SUS $)=20,75-20,34\left(1-\mathrm{e}^{-6,96 x}\right)$} & 1,00 \\
\hline \multicolumn{2}{|c|}{$y($ IMA-RES $)=57,37\left(1-\mathrm{e}^{-1,23 x}\right)$} & 0,84 & y ( & $A-R E S)=16,25$ & $2,40\left(1-e^{-0,16 x}\right)$ & 0,89 \\
\hline y (IMA & $61\left(1-e^{-7,39 x}\right)$ & 1,00 & y ( & A-SUS) $=15,45$ & $4,13\left(1-e^{-9,86 x}\right)$ & 1,00 \\
\hline
\end{tabular}


derando-se os valores exponenciais do modelo, verificou-se que o biótipo resistente apresentou valor de MSPA aproximadamente 62 vezes maior, em relação ao mesmo valor apresentado pelo biótipo susceptível. Esses resultados corroboram os observados para a variável controle, em relação à qual o biótipo resistente apresentou menor resposta às doses iniciais testadas.

Os resultados obtidos permitem inferir que o biótipo de arroz-vermelho ORYSA 184 é resistente ao herbicida imazapyr + imazapic. Foi possível observar, também, a inexistência de resistência do biótipo ORYSA 184 aos herbicidas clethodim e glyphosate e confirmar a susceptibilidade do biótipo ORYSA 188 a todos os herbicidas testados. Resultados semelhantes foram observados para Cyperus difformis (Agostinetto et al., 2011) e Bidens subalternans (Lamego, 2008), resistentes a herbicidas inibidores de ALS, cujo controle satisfatório foi obtido com herbicidas de mecanismos de ação distintos.

A ocorrência de arroz-vermelho resistente tende a evoluir, em decorrência de fluxo gênico entre a planta daninha e o arroz cultivado resistente (Sales et al., 2008), pois, embora o arroz-vermelho seja planta autógama, a taxa de fecundação cruzada pode variar de 0,065 (Villa et al., 2006) até 0,25\% (Shivrain et al., 2008). Por essa razão, considerando-se que a resistên- cia do arroz-vermelho, aos herbicidas inibidores da ALS, caracteriza-se por apresentar alelo dominante e ser transmitida pelo pólen (Vidal \& Fleck, 1997), há, como consequência, a rápida perda da possibilidade da utilização de herbicida para controle de arroz-vermelho, a qual, hoje, é a principal forma de controle dessa planta daninha em lavouras de arroz irrigado.

No segundo experimento, para todas as variáveis, observou-se interação dos fatores testados (Tabelas 2 e 3). O herbicida imazapyr + imazapic apresentou menor eficiência de controle do biótipo resistente de arrozvermelho (ORYSA 184), em comparação com a de seu controle do biótipo susceptível, em todas as doses testadas (Tabela 2).

Com relação à MSPA, o biótipo susceptível apresentou redução na produção da variável em relação ao biótipo resistente, com exceção das duas maiores doses testadas (Tabela 2). Não evidenciam diferenças as produções de MSPA pelas plantas do biótipo susceptível e pelas do biótipo resistente que não receberam tratamento herbicida.

$\mathrm{A} \mathrm{C}_{50}$ de imazapyr + imazapic, para o biótipo susceptível, foi inferior a $25 \%$ da dose comercial, demonstrando elevada eficácia para esse biótipo, enquanto, para o biótipo resistente foram necessários $200 \%$ da dose comercial,

Tabela 2. Controle (\%) e matéria seca da parte aérea - MSPA (g) de biótipos de arroz-vermelho resistente (RES) e susceptível (SUS) ao herbicida imazapyr + imazapic, em função da aplicação de diferentes doses do herbicida imazapyr + imazapic, avaliado aos 28 dias após aplicação dos tratamentos. FAEM/UFPel, Capão do Leão/RS, 2010/11

\begin{tabular}{|c|c|c|c|c|c|c|c|c|c|}
\hline \multirow{2}{*}{ Biótipo } & \multicolumn{9}{|c|}{ Dose $^{1}$} \\
\hline & 0 & 0,5 & 1 & 2 & 4 & 8 & 16 & 32 & 64 \\
\hline \multicolumn{10}{|c|}{ Controle (\%) } \\
\hline Resistente & 0,00 ns & $22,0 *$ & $42,2 *$ & $49,5^{*}$ & $36,3^{*}$ & $64,5^{*}$ & $68,2 *$ & $64,2 *$ & $87,2 *$ \\
\hline Susceptível & 0,0 & 96,2 & 96,7 & 97,2 & 98,7 & 99,2 & 99,5 & 100,0 & 100,0 \\
\hline \multicolumn{10}{|c|}{$\operatorname{MSPA}(\mathrm{g})$} \\
\hline Resistente & $17,84^{\mathrm{ns}}$ & $15,69 *$ & $12,88 *$ & $10,36^{*}$ & $13,06 *$ & $6,79 *$ & $5,47 *$ & $3,47^{\text {ns }}$ & $1,20^{\mathrm{ns}}$ \\
\hline Susceptível & 15,45 & 1,43 & 1,40 & 1,64 & 1,20 & 1,27 & 1,26 & 1,02 & 0,98 \\
\hline \multicolumn{2}{|c|}{ Equações (Controle) $^{2}$} & \multicolumn{2}{|c|}{$\mathbf{R}^{2}$} & & \multicolumn{3}{|c|}{ Equações (MSPA) } & & $\mathbf{R}^{2}$ \\
\hline \multirow{2}{*}{\multicolumn{2}{|c|}{$\begin{array}{l}y(\operatorname{RES})=65,33\left(1-\mathrm{e}^{-0,76 x}\right) \\
y(\text { SUS })=98,81\left(1-\mathrm{e}^{-7,22 x}\right)\end{array}$}} & \multicolumn{2}{|c|}{0,65} & & \multicolumn{4}{|c|}{$\mathrm{y}(\mathrm{RES})=15,91-13,60\left(1-\mathrm{e}^{-0,11 x}\right)$} & 0,84 \\
\hline & & \multicolumn{2}{|c|}{0,99} & & \multicolumn{4}{|c|}{$y($ SUS $)=15,45-14,18\left(1-\mathrm{e}^{-9,04 x}\right)$} & 0,96 \\
\hline
\end{tabular}

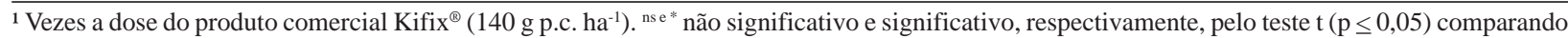
os biótipos. ${ }^{2}$ Modelos ajustados a partir das interações e seus respectivos coeficientes de determinação $\left(\mathrm{R}^{2}\right)$.

Tabela 3. Doses do herbicida imazapyr + imazapic (vezes a dose recomendada) necessárias para controlar 50\% da população de plantas $\left(\mathrm{C}_{50}\right)$ e reduzir $50 \%$ da produção de matéria seca da parte aérea $\left(\mathrm{MS}_{50}\right)$ do biótipo resistente $(\mathrm{R})$ e susceptível (S) de arrozvermelho e os respectivos fatores de resistência (FR). FAEM/UFPel, Capão do Leão/RS, 2010/11

\begin{tabular}{lccc}
\hline & \multicolumn{3}{c}{ Biótipo } \\
\cline { 2 - 4 } & Resistente & Susceptível & FR $^{\mathbf{3}}$ \\
\hline $\mathrm{C}_{50}(\mathrm{x} \text { a dose recomendada })^{1}$ & 2,0 & 0,1 & 20 \\
$\mathrm{MS}_{50}(\mathrm{x} \text { a dose recomendada })^{2}$ & 7,0 & 0,1 & 70 \\
\hline
\end{tabular}

${ }^{1}$ Dados obtidos pela equação exponencial y $=a\left(1-e^{-b x}\right),(\mathrm{p} £ 0,05) ;{ }^{2}$ Dados obtidos pela equação exponencial y $=\mathrm{y}_{0}+a\left(1-e^{-b x}\right),(\mathrm{p} 0,05)$; ${ }^{3}$ Fator de resistência $=\mathrm{C}_{50} \mathrm{R} / \mathrm{C}_{50} \mathrm{~S}$ e $\mathrm{MS}_{50} \mathrm{R} / \mathrm{MS}_{50} \mathrm{~S}$, respectivamente. 
sendo observado FR de 20, ou seja, o biótipo resistente é 20 vezes menos sensível ao imazapyr + imazapic do que o biótipo susceptível (Tabela 3). Em comparação, estudo realizado com $C$. difformis demonstrou $\mathrm{C}_{50}$ de $600 \%$ da dose recomendada do herbicida pyrazosulfuron-ethyl para o biótipo resistente, porém, quando utilizado bispyribacsodium a $\mathrm{C}_{50}$ foi muito próxima da dose recomendada do produto (Galon et al., 2008).

A $\mathrm{MS}_{50}$ para o biótipo resistente foi de 7 vezes a dose de registro, enquanto para o biótipo susceptível foi de 0,1 vez a dose de registro (Tabela 3). Esses resultados confirmam os observados no primeiro experimento, no qual o biótipo ORYSA 184 apresentou resistência a imazapyr + imazapic. O FR foi de 70, ou seja, para o biótipo resistente é necessária dose 70 vezes superior para obtenção de resultado similar ao obtido para o biótipo susceptível.

$\mathrm{O} \mathrm{FR}$, com base na $\mathrm{MS}_{50}$, foi superior àqueles apresentados na literatura para herbicidas inibidores de ALS, na qual os valores encontrados foram de 18,8 e 20,5 para Parthenium hysterophorus resistente a cloransulammethyl e chlorimuron-ethyl, respectivamente (Gazziero et al., 2006), e de 22,1 e 12,3, para B. subalternans resistente a chlorimuron-ethyl e imazethapyr, respectivamente (López-Ovejero et al., 2006). Isso mostra a grande variação da resistência aos herbicidas inibidores da ALS, entre diferentes plantas daninhas.

Com o objetivo de estimar com maior precisão o FR, foi conduzido o experimento III, pois, no experimento anterior (II), a $\mathrm{C}_{50}$ e a $\mathrm{MS}_{50}$ ficaram abaixo da menor dose herbicida testada para biótipo susceptível. Neste experimento, também foi constatada interação dos fatores biótipo e dose, para todas as variáveis. O controle do arroz-vermelho pelo herbicida imazapyr + imazapic ajustou-se à equação de regressão sigmoidal do tipo logístico, para todas as variáveis, apresentando ajuste satisfatório dos dados ao modelo (Figura 1).

O herbicida imazapyr + imazapic apresentou menor eficiência de controle do biótipo resistente de arroz-vermelho (ORYSA 184), em comparação com a eficiência de controle do biótipo susceptível, nas doses passíveis de comparação, ou seja, até quatro vezes a dose recomendada do produto (Figura $1 \mathrm{~A}$ ). O herbicida imazapyr + imazapic demonstrou, novamente, controle eficiente das plantas do

A

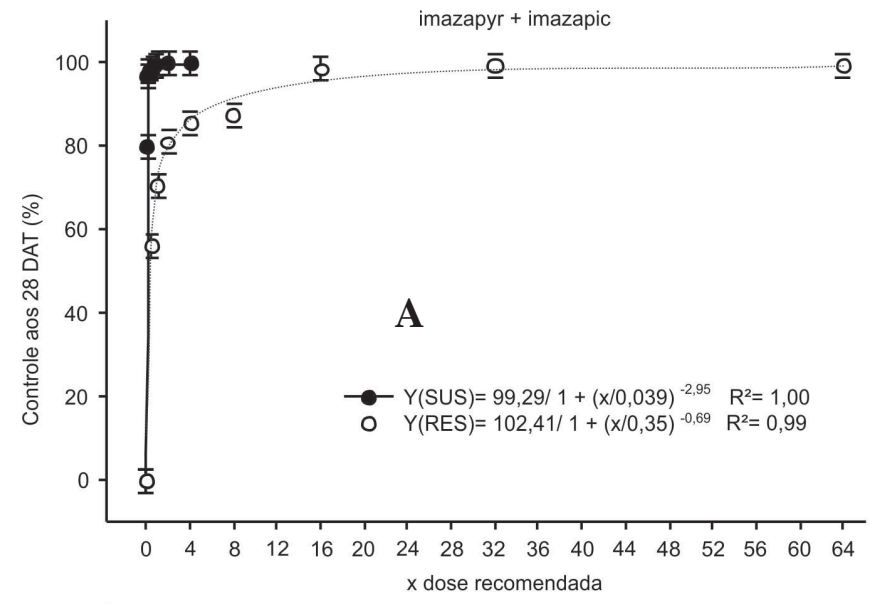

B

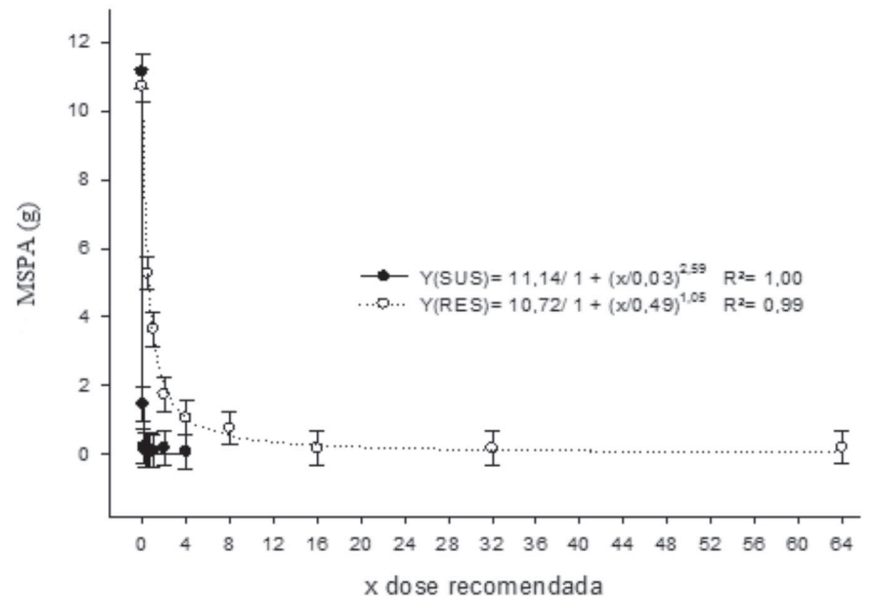

Figura 1. Controle (\%) (A) e matéria seca da parte aérea - MSPA (g) (B) de biótipos de arroz-vermelho resistente (RES) e susceptível (SUS) ao herbicida imazapyr + imazapic, pelo herbicida imazapyr + imazapic, aos 28 dias após a aplicação dos tratamentos (DAT). FAEM/UFPel, Capão do Leão/RS, 2011/12. Obs: As barras verticais representam os intervalos de confiança a $95 \%$. 
biótipo susceptível, em todas as doses herbicidas. Em contrapartida, o biótipo resistente não foi controlado, corroborando o verificado no experimento II.

Com relação à MSPA, o biótipo susceptível apresentou redução na produção da variável, em relação ao biótipo resistente, nas doses de 0,5 ; 1 e 2 vezes a dose de registro (Figura 1 B). Não foram observadas diferenças de produção de MSPA entre plantas dos biótipos susceptível e resistente que não receberam tratamento herbicida, corroborando os resultados encontrados no experimento II. Para essa variável, MSPA, verificou-se drástica redução da sua produção pelo biótipo susceptível, mesmo nas doses mais baixas, enquanto, para o biótipo resistente, a redução na produção de MSPA ocorreu em doses mais elevadas.

Com base na ausência de sobreposição do intervalo de confiança (IC) do biótipo susceptível em relação ao resistente, foi possível estabelecer o FR. Os valores de $\mathrm{FR}$, com base na $\mathrm{C}_{50} \mathrm{e} \mathrm{MS}_{50}$ foram de 8,97 e 16,33, respectivamente, representando o número de vezes que a dose do herbicida imazapyr + imazapic deve ser aumentada para promover 50\% de controle ou de redução da MSPA do biótipo resistente em relação à redução ou controle do biótipo susceptível (Tabela 4).

Considerando-se o valor de FR encontrado, tanto com base na $\mathrm{C}_{50}$ quanto na $\mathrm{MS}_{50}$, o biótipo de arroz-vermelho ORYSA 184 demonstrou novamente, ser resistente ao herbicida imazapyr + imazapic (Tabela 4), embora os va- lores dos FRs, relacionados com a $\mathrm{C}_{50}$ ou com a $\mathrm{MS}_{50}$, tenham sido inferiores aos encontrados no experimento II (Tabela 3). Em estudo realizado com $C$. difformis resistente ao herbicida pyrazosulfuron-ethyl, o FR encontrado com base no controle foi superior a 103,2 (Agostinetto et al., 2011), superando o verificado neste estudo.

Para arroz-vermelho foi observado FR relacionado com a $\mathrm{C}_{50}$ de 9,3; 7,8 e 6,5 entre os biótipos testados, para o herbicida imazethapyr (Dornelles et al., 2010), valores inferiores aos encontrados nos experimentos realizados. Resultados inferiores também foram encontrados com este herbicida em relação ao $\mathrm{MS}_{50}$, em que os FRs para arroz-vermelho resistente variaram de 2,3 (Dornelles et al., 2010) a 11,6 (Menezes et al., 2009). Os elevados FRs encontrados estão relacionados com o biótipo utilizado, o qual é oriundo de área onde o cultivo do arroz é intenso e a utilização de herbicidas é a principal forma de controle de arroz-vermelho, ocasionando a seleção destas populações resistentes.

Os valores de $\mathrm{C}_{50}$ e $\mathrm{MS}_{50}$ para o biótipo susceptível, mesmo com a redução das doses testadas, foram inferiores à menor dose utilizada (equivalente à $0,0625 \mathrm{vez}$ a dose de registro do produto). Desta forma, observou-se elevada susceptibilidade do biótipo de arroz-vermelho ORYSA 188 ao herbicida imazapyr + imazapic. Este fato decorre de o herbicida nunca ter sido utilizado na área de coleta do biótipo.

Tabela 4. Valores de $\mathrm{C}_{50}$ e $\mathrm{MS}_{50}$ com intervalos de confiança (IC) e fator de resistência do biótipo resistente e susceptível, em resposta à aplicação de diferentes doses do herbicida imazapyr + imazapic, avaliado aos 28 DAT. UFPel, Capão do Leão - RS, 2010/11

\begin{tabular}{|c|c|c|c|}
\hline \multirow{2}{*}{ Biótipo } & $\mathrm{x}$ dose comercial & $95 \%$ IC & \multirow{2}{*}{ Fator de resistência ${ }^{3}$} \\
\hline & \multicolumn{2}{|c|}{$\mathrm{C}_{50}{ }^{1}$} & \\
\hline Resistente & 0,350 & $0,287-0,391$ & \multirow[t]{2}{*}{8,97} \\
\hline Susceptível & 0,039 & $0,038-0,041$ & \\
\hline \multicolumn{4}{|c|}{$\mathrm{MS}_{50}{ }^{2}$} \\
\hline Resistente & 0,490 & $0,459-0,521$ & 16,33 \\
\hline Susceptível & 0,030 & $-0,018-0,083$ & \\
\hline
\end{tabular}

${ }^{1} \mathrm{C}_{50}=$ dose necessária para obter $50 \%$ de controle; ${ }^{2} \mathrm{MS}_{50}=$ dose necessária para obter $50 \%$ de redução da matéria da parte aérea seca; ${ }^{3}$ Fator de resistência ao herbicida imazapyr + imazapic dos biótipos de arroz-vermelho, obtido da divisão da $\mathrm{C}_{50}$ ou $\mathrm{MS}_{50}$ do biótipo resistente em relação ao biótipo susceptível ao imazapyr + imazapic.

\section{CONCLUSÕES}

O biótipo ORYSA 184 é resistente ao imazapyr + imazapic, quando aplicada a dose máxima de registro no estádio de desenvolvimento da planta daninha indicado.

Os herbicidas clethodim e glyphosate, com mecanismos de ação alternativos ao imazapyr + imazapic, controlam o biótipo resistente ORYSA 184 de arroz-vermelho.

O controle do biótipo ORYSA 184 é inviável com o herbicida imazapyr + imazapic.

\section{REFERÊNCIAS}

Agostinetto D, Dal Magro T, Vargas L \& Noldin JA (2011) Resistência de Cyperus difformis L. ao herbicida pyrazosulfuron-ethyl e alternativas de controle. Semina, Ciências Agrárias, 32:839-848.

Agrofit (2012) Sistema de Agrotóxicos Fitossanitários. Ministério da Agricultura, Pecuária e Abastecimento, Coordenação Geral de Agrotóxicos e Afins. Disponível em: <http:// agrofit.agricultura.gov.br/agrofit_cons/principal_agrofit_cons>. Acessado em: 14 de outubro 2012.

Christoffoleti PJ (2002) Curvas de dose-resposta de biótipos resistente e susceptível de Bidens pilosa L. aos herbicidas inibidores da ALS. Scientia Agricola, 59:513-519. 
Croughan TP, Utomo HS, Sanders DE \& Braveman MP(1996) Herbicideresistant rice offers potential solution to red rice problem. Los Angeles Agricultural, 39:10-12.

Dornelles SHB, Garcia DC, Loreto EL, Canto-Dorow TS, Manfron PA, Sanchotene DM \& Oliveira LFV (2010) Suscetibilidade de biótipos de arroz-vermelho e de cultivares de arroz irrigado ao herbicida imazethapyr. Planta Daninha, 28:1097-1106.

Embrapa - Centro Nacional de Pesquisa de Solos (CNPS) (2006) Sistema Brasileiro de Classificação de Solos. $2^{a}$ ed. Rio de Janeiro, Embrapa Solos. 412p.

Fleck NG, Agostinetto D, Galon L \& Schaedler CE (2008) Competitividade relativa entre cultivares de arroz irrigado e biótipo de arroz-vermelho. Planta Daninha, 26:101-111.

Galon L, Panozzo LE, Noldin JA, Concenço G, Tarouco CP, Ferreira EA, Agostinetto D, Silva AA \& Ferreira FA (2008) Resistência de Cyperus difformis a herbicidas inibidores da ALS em lavoura de arroz irrigado em Santa Catarina. Planta Daninha, 26:419-427.

Gazziero DLP, Brighenti AM \& Voll E (2006) Resistência cruzada da losnabranca (Parthenium hysterophorus) aos herbicidas inibidores da enzima acetolactato sintase. Planta Daninha, 24:157-162.

Hall LM, Strome KM, Mallory-Smith CA \& Thill DC (1998) Resistence to acetolactate sintase inhibitors and quinclorac in a biotypes of false clover (Gallium sourium). Weed Science, 46:390-396.

Lamego FP (2008) Elucidação do mecanismo de resistência aos herbicidas inibidores de ALS na espécie poliplóide Bidens subalternans DC. Tese de Doutorado. Porto Alegre, Universidade Federal do Rio Grande do Sul. 152p.

Lamego FP, Charlson D, Delatorre CA, Burgos NR \& Vidal RA (2009) Molecular basis of resistance to ALS-inhibitor herbicides in greater beggarticks. Weed Science, 57:474-481.

López-Ovejero RF, Carvalho SJP, Nicolai M, Abreu AG, GromboneGuaratini MT, Toledo REB \& Christoffoleti PJ (2006) Resistance and differential susceptibility of Bidens pilosa and B. subalternans biotypes to ALS-inhibiting herbicides. Scientia Agricola, 63:139-145.
Menezes VG, Mariot CHP, Kalsing A \& Goulart ICGR (2009) Arroz-vermelho (Oryza sativa) resistente aos herbicidas imidazolinonas. Planta Daninha, 27:1047-1052.

Rizzardi MA, Vargas L, Roman ES \& Kissman K (2004) Aspectos gerais do controle de plantas. In: Vargas L \& Roman ES (Eds) Manual de manejo e controle de plantas daninhas. Bento Gonçalves, Embrapa Uva e Vinho. p.105-144.

Sales MA, Shivrain VK, Burgos NR \& Kuk YI (2008) Amino acid substitutions in the acetolactate synthase gene of red rice (Oryza sativa) confer resistance to imazethapyr. Weed Science, 56:485489.

Seefeldt SS, Jensen JE \& Fuerst EP (1995) Log-logistic analysis of herbicide dose-response relationships. Weed Technology, 9:218-227.

Shivrain VK, Burgos NR, Gealy DR, Moldenhauer KAK \& Baquireza CJ (2008) Maximum outcrossing rate and genetic compatibility between red rice (Oryza sativa) biotypes and Clearfield ${ }^{\mathrm{TM}}$ rice. Weed Science, 56:807-813.

Shivrain VK, Burgos NR, Gealy DR, Smith KL, Scott RC, Mauromoustakos A \& Black H (2009) Red rice (Oryza sativa) emergence characteristics and influence on rice yield at different planting dates. Weed Science, 57:94-102.

Steele GL, Chandler JM \& McCauley GN (2002) Control of red rice (Oryza sativa) in imidazolinone-tolerant rice (Oryza sativa). Weed Technology, 16:627-630.

Vidal RA \& Fleck NG (1997) Análise do risco da ocorrência de biótipos de plantas daninhas resistentes aos herbicidas. Planta Daninha, 15:152-161.

Vidal RA \& Merotto Júnior A (2001) Herbicidologia. $1^{\mathrm{a}}$ ed. Porto Alegre, Evangraf. 152p.

Villa SCC, Marchezan E, Avila LA, Massoni PFS, Telo GM, Machado SLO \& Camargo ER (2006) Arroz tolerante a imidazolinonas: controle do arroz-vermelho, fluxo gênico e efeito residual do herbicida em culturas sucessoras não-tolerantes. Planta Daninha, 24:761-768. 